\title{
Combining paratransgenesis with SIT: impact of ionizing radiation on the DNA copy number of Sodalis glossinidius in tsetse flies
}

Güler Demirbas-Uzel ${ }^{1,2}$, Linda De Vooght ${ }^{3}$, Andrew G. Parker', Marc J. B. Vreysen', Robert L. Mach³, Jan Van Den Abbeele ${ }^{3}$ and Adly M. M. Abd-Alla ${ }^{1^{*}}$

\begin{abstract}
Background: Tsetse flies (Diptera: Glossinidae) are the cyclical vectors of the causative agents of African Trypanosomosis, which has been identified as a neglected tropical disease in both humans and animals in many regions of sub-Saharan Africa. The sterile insect technique (SIT) has shown to be a powerful method to manage tsetse fly populations when used in the frame of an area-wide integrated pest management (AW-IPM) program. To date, the release of sterile males to manage tsetse fly populations has only been implemented in areas to reduce transmission of animal African Trypanosomosis (AAT). The implementation of the SIT in areas with Human African Trypanosomosis (HAT) would require additional measures to eliminate the potential risk associated with the release of sterile males that require blood meals to survive and hence, might contribute to disease transmission. Paratransgenesis offers the potential to develop tsetse flies that are refractory to trypanosome infection by modifying their associated bacteria (Sodalis glossinidius) here after referred to as Sodalis. Here we assessed the feasibility of combining the paratransgenesis approach with SIT by analyzing the impact of ionizing radiation on the copy number of Sodalis and the vectorial capacity of sterilized tsetse males.

Results: Adult Glossina morsitans morsitans that emerged from puparia irradiated on day 22 post larviposition did not show a significant decline in Sodalis copy number as compared with non-irradiated flies. Conversely, the Sodalis copy number was significantly reduced in adults that emerged from puparia irradiated on day 29 post larviposition and in adults irradiated on day 7 post emergence. Moreover, irradiating 22-day old puparia reduced the copy number of Wolbachia and Wigglesworthia in emerged adults as compared with non-irradiated controls, but the radiation treatment had no significant impact on the vectorial competence of the flies.

Conclusion: Although the radiation treatment significantly reduced the copy number of some tsetse fly symbionts, the copy number of Sodalis recovered with time in flies irradiated as 22-day old puparia. This recovery offers the opportunity to combine a paratransgenesis approach - using modified Sodalis to produce males refractory to trypanosome infection - with the release of sterile males to minimize the risk of disease transmission, especially in HAT endemic areas. Moreover, irradiation did not increase the vector competence of the flies for trypanosomes.
\end{abstract}

Keywords: Symbiont, Glossinidae, Trypanosomosis, Trypanosomiasis, Glossina morsitans morsitans

\footnotetext{
* Correspondence: a.m.m.abd-alla@iaea.org

${ }^{1}$ Insect Pest Control Laboratory, Joint FAO/IAEA Division of Nuclear

Techniques in Food and Agriculture, Vienna International Centre, P.O. Box

100, 1400 Vienna, Austria

Full list of author information is available at the end of the article
}

(C) International Atomic Energy Agency; licensee BioMed Central Ltd. 2018 This is an open access article distributed under the terms of the Creative Commons Attribution IGO License (https://creativecommons.org/licenses/by/3.0/igo/) which permits unrestricted use, distribution, and reproduction in any medium, provided appropriate credit to the original author(s) and the source is given. 


\section{Background}

Tsetse flies (Glossina spp., Diptera: Glossinidae) are the cyclical vectors of African trypanosomes, which cause a devastating and economically important infectious disease; sleeping sickness or Human African Trypanosomosis (HAT) in humans and nagana or Animal African Trypanosomosis (AAT) in livestock. Nagana causes high mortality in livestock and HAT is a serious health burden and risk to 60 million people in endemic regions of 36 countries in sub-Saharan Africa [1, 2]. Trypanosoma vivax, $T$. congolense and T. brucei brucei are the major tsetse transmitted pathogens in livestock [3] while T. brucei rhodesiense and T. brucei gambiense cause sleeping sickness in humans [4]. Members of the morsitans and palpalis groups of Glossina are efficient vectors of HAT and AAT [5]. In the absence of vaccines and efficient, safe and inexpensive drugs $[6,7]$, combined with increasing resistance against the current trypanocidal drugs $[6,8]$, control of the insect vector remains an essential part of managing disease transmission [9-11]. Most of the vector control strategies are insecticide-based $[12,13]$.

The sterile insect technique (SIT) is a species-specific, safe, efficient, environment friendly, biological-based control tactic to manage populations (suppression or/and elimination) of insect pests and disease vectors [14]. The SIT entails mass-rearing the target insects, sterilization of the males using ionizing radiation and sequential area-wide release of a large number of sterile males into the target area. The sterile flies compete for mating with the female wild population, interrupting their reproductive potential ultimately resulting in population reduction or elimination $[15,16]$.

It is crucial that when large numbers of male vectors are released their ability to transmit pathogens should be curtailed to the maximum possible extent. In past and current tsetse fly programs that had an SIT component, sterile males received two blood meals supplemented with the trypanocidal drug isometamidum chloride $(10 \mu \mathrm{g} / \mathrm{ml})$ before their release to minimize the risk of disease transmission. This treatment blocks the transmission ability of flies for $T . b$. brucei and reduces the transmission ability of flies for $T$. congolense by 5 -fold under laboratory conditions [17]. However, a field study demonstrated that the use of this treatment was not entirely sufficient to prevent sterile males of Glossina palpalis gambiensis from transmitting the trypanosomes $T$. congolense and T. vivax [18]. Therefore, the development of tsetse fly strains refractory to trypanosome transmission would significantly contribute to the applicability of the SIT for the management of tsetse flies, especially in HAT endemic areas.

Tsetse flies harbor four main symbiotic microbes; Wigglesworthia, Sodalis, Wolbachia [19] and the recently found Spiroplasma [20]. The primary mutualist Wigglesworthia resides intracellularly in mycetocytes within the mycetome as well as extracellularly within maternal milk gland secretions. It provides dietary supplements that are necessary for host fecundity and is involved in the maturation process of the adult immune system [21]. In the absence of Wigglesworthia in the larvae, subsequent adults are characterized by an underdeveloped cellular immune system and exhibit unusual susceptibility for trypanosome infections and are sterile [21-25]. The facultative symbiont Sodalis displays a wide tissue tropism and is present both intra- and extracellularly in the tsetse fly midgut, muscle, fat body, milk glands, and salivary glands. The functional role of Sodalis in tsetse flies is relatively unknown although its influence on host longevity and modulation of susceptibility to trypanosome infection has been reported [26-28]. While all individuals in laboratory colonies harbor Sodalis, infection in natural populations varies in different species analyzed [29, 30]. The third symbiont, Wolbachia is an alpha-proteobacterium, located intracellularly in tsetse germ line tissues and is involved in cytoplasmic incompatibility. Wolbachia can be found in natural populations of tsetse flies with a prevalence varying between 0 and 100\% depending on the species [31, 32]. Finally, Spiroplasma is a genus of wall-free motile, gram-positive bacteria [33, 34] associated both intracellularly and extracellularly in a variety of arthropods. It was recently identified as a novel symbiont of $G$. $f$. fuscipes and G. tachinoides [20].

Symbiotic microbes in tsetse flies have a vital role due to their significant influence on the biology of the fly, its reproduction, immunity, elicitation of phenotypes and potential effects on their vector competence for trypanosomes [35-38]. Understanding the interactions of the symbionts and parasites occuring in tsetse hosts might facilitate the development of tsetse flies refractory to trypanosome infection by modifying their symbionts. Paratransgenesis is a new genetic method based on modifying symbiotic organisms of insect vectors using recombinant technologies to express effector molecules, including ones that can potentially block pathogen development [39, 40]. As trypanosomes develop in the midgut, proventriculus and salivary glands of tsetse flies, foreign gene products need to be expressed in at least one of those tissues $[23,41]$. Sodalis is an ideal candidate for paratransgenesis due to its presence in the midgut and the fact that it is one of the few insect bacterial symbionts that can be cultured and genetically modified in vitro [5, 42-44]. Sodalis has been genetically engineered to express and release significant amounts of functional anti-trypanosome nanobodies in different tissues of the tsetse fly [45]. A crucial step in implementing paratransgenesis in tsetse flies for use in SIT programs is the stable colonization of sterile male flies with recombinant Sodalis strains expressing trypanosome-interfering proteins. However, the impact of ionizing radiation on tsetse symbionts, especially Sodalis, is unknown. 
The recent demonstration of tsetse pupae sex separation using near infrared imaging several days before adult emergence from the puparium [46] opens the possibility of irradiating males in the puparial stage. We investigated the impact of ionizing radiation treatment conducted at three different life stages on Sodalis copy number in adult G. m. morsitans flies at different times post emergence. Although the tsetse fly males are the sex of interest for SIT programmes, the impact of radiation on Sodalis copy number in females was also investigated as this effect has not been analyzed previously. After determining the optimum development phase for irradiation, i.e. having the least effect on Sodalis copy number, we tested the impact of irradiation on the male's vector competence for trypanosomes as well as the impact on Wigglesworthia, and Wolbachia. We discuss the significance of our findings in the context of improving the application of SIT and paratransgenesis to manage tsetse fly populations and hence to control African trypanosomosis.

\section{Methods}

\section{Tsetse fly}

The colony of the tsetse fly G. m. morsitans used in this study originated from Zimbabwe and has been maintained at the Insect Pest Control Laboratory (IPCL) of the Joint FAO/IAEA Division of Nuclear Techniques in Food and Agriculture, Seibersdorf, Austria since 1997. The colony and experimental flies were maintained at $24 \pm 0.5^{\circ} \mathrm{C}$ and $75-80 \% \mathrm{RH}$ and were fed on defibrinated bovine blood (Svaman spol s.r.o., Majava, Slovakia) using the artificial (in vitro) membrane feeding system for 1520 min three times per week [47, 48].

\section{Analysis of the dynamics of Sodalis copy number in a $G$. m. morsitans colony}

To assess the dynamics of Sodalis copy number in the G. m. morsitans colony established under laboratory rearing condition, samples of 4 males and 4 females were taken on day $0,1,2,3,4,5,6,7,14,21$ and 30 post emergence. Samples were placed at $-20{ }^{\circ} \mathrm{C}$ until DNA extraction.

\section{Experimental design}

The impact of gamma irradiation was determined on both females and males. They were irradiated at three developmental stages to assess the effect on the copy number of Sodalis, Wigglesworthia and Wolbachia: (a) 7-day old adults, (b) 29-day old puparia (36 $\pm 12 \mathrm{~h}$ before emergence), (c) 22-day old puparia. Teneral tsetse males that emerged from puparia irradiated with 110 Gy on day 22 post larviposition were tested for vector competence for trypanosomes.

\section{Irradiation procedures}

The tsetse puparia and adults were irradiated in air at the IPCL, Seibersdorf, Austria using a ${ }^{60} \mathrm{Co}$ Gammacell ${ }^{\circ}$ 220 (MDS Nordion Ltd., Ottawa, Canada). The dose rate was measured by alanine dosimetry as $2.144 \mathrm{~Gy} \cdot \mathrm{sec}^{-1}$ on 2015-03-03 with an expanded uncertainty $(k=2)$ of $3.2 \%$. The radiation field was mapped using Gafchromic HD-V2 film and the dose uniformity ratio in the volume used for the experiments was $<1.2$ for adult exposures and $<1.1$ for pupal exposures. The desired radiation doses were given by varying the time of exposure of the samples to give minimum doses of 20, 50 and 110 Gy (the dose currently used in tsetse SIT programs). Untreated puparia or flies were used as control (0 Gy) and handled in the same manner. For adults, 7-day old flies (males and females) were placed in small cages $(11 \mathrm{~cm}$ diameter $\times 4.5 \mathrm{~cm}$ height $)$ and placed in the center of the chamber for treatment. The pupae were placed in plastic Petri-dishes (diameter $5.5 \mathrm{~cm}$, height $1.5 \mathrm{~cm}$ ) that allowed irradiation in the center of the chamber.

In the first part, 7-day old males and females that had already been maintained under the above-mentioned colony conditions and offered three normal blood meals were irradiated at $48 \mathrm{~h}$ post the last blood meal with 20,50 and 110 Gy at a density of 72 flies per cage with two replicates. After irradiation, all emerged flies of the different treatments and the control groups were held under standard insect rearing conditions and were offered normal blood meals every other day of the week during the length of the experiment. Four females and 4 males were frozen for each dose on day $0,1,7$ and 14 post-irradiation. For the day 0 group, both females and males were frozen approximately $20 \mathrm{~min}$ after irradiation. All frozen samples were kept at $-20{ }^{\circ} \mathrm{C}$ until being used for further analysis. The experiment was replicated twice.

In the second and third part of the study, batches of puparia were collected on the same day on day 22- and 29 post larviposition. Collected puparia for each radiation dose were kept in Petri dishes and exposed to 20, 50 and $110 \mathrm{~Gy}$. The experiment was replicated two and three times for 22- and 29-day old puparia, respectively. Irradiated and non-irradiated pupae were kept under standard colony conditions. Daily examinations were made for fly emergence, and non-emerged puparia were observed for each treatment. Emerged flies were collected daily and transferred to standard fly holding cages (20 cm diameter $\times 5 \mathrm{~cm}$ height) at a density of 72 flies per cage. Emerged female and male flies were held in separate holding cages during the experiment. Four females and 4 males were frozen on day $0,1,3,5,7$ and 14 post emergence separately for each dose and kept at $-20{ }^{\circ} \mathrm{C}$ until further analysis. 


\section{DNA extraction and quantitative PCR}

The total DNA of each individual fly was extracted from the collected flies using the DNeasy tissue kit (QIAGEN Inc., Valencia, CA) following the manufacturer's instructions. The extracted DNA was eluted in $200 \mu \mathrm{l}$ elution buffer and DNA extracts from individual samples were pooled (4 females and 4 males, separately). The pooled DNA concentration was measured by spectrophotometry (Nanodrop-Synergy H1 Multi-Mode Reader, BioTek, Instruments, Inc., USA). All DNA samples were diluted to a final concentration of $4 \mathrm{ng} / \mu \mathrm{l}$ and $5 \mu \mathrm{l}$ of the diluted DNA was used for qPCR to determine symbiont DNA copy number as previously described $[49,50]$. The tsetse reference gene $\beta$-tubulin was used to normalize the qPCR reactions [50]. Sodalis, Wigglesworthia and Wolbachia densities were quantified for both sexes at different days post irradiation/emergence for all treatments by qPCR using primers that target the fliC, codhoc and Wolbachia 16S rRNA genes, respectively. The primers and the PCR conditions are given in Additional file 1.

\section{Tsetse fly infection with trypanosomes, maintenance, and} dissection

For the infection experiment, teneral flies emerged from 22-day old irradiated (110 Gy) and non-irradiated puparia (collected and irradiated at the IPCL and shipped to the Unit of Veterinary Protozoology, Institute of Tropical Medicine (ITM), Antwerp, Belgium) were offered a blood meal containing a highly transmissible pleiomorphic $T$. brucei brucei (Tbb) AnTAR1 strain, $24 \mathrm{~h}$ after emergence. $T b b$ AnTAR1 is a post-tsetse fly strain derived from the EATRO 1125 stabilate that was originally isolated from a bushbuck in Uganda in 1966 [51]. Parasitized blood was harvested with heparin from cyclophosphamide-immune suppressed mice (Endoxan ${ }^{\circ}$, Baxter) 6 days post-infection and mixed with defibrinated horse blood (E\&O Laboratories) to obtain $>10^{6}$ bloodstream form (BSF) trypanosomes $/ \mathrm{ml}$ with $80 \%$ intermediate/stumpy forms in the infectious blood meal. This tsetse-trypanosome infection model has given good infection rates in the midgut and salivary glands of tsetse flies [52]. Flies that did not take the infectious blood meal were excluded from the experiment. Subsequently, the remaining flies were maintained for 4 weeks at $26 \pm 0.5{ }^{\circ} \mathrm{C}$ and $65 \pm 5 \%$ relative humidity and offered uninfected defibrinated horse blood three times per week using an artificial membrane feeding system [47]. Twenty eight days after the infective blood meal, individual flies were analyzed for the presence of procyclic and metacyclic trypanosomes (the reproductive and transmissible forms) by microscopical examination of their midguts and salivary glands, respectively. Differences in infection rates between irradiated and control flies were compared using Fisher's exact test (two-sided) and considered significant if $P$-values were lower than 0.05 .

\section{Statistical analysis}

The statistical analysis and graphics were executed in $\mathrm{R}$ [53] using RStudio version 3.4.1. [54] with the packages ggplot2 v2.2.1 [55], lattice v0.20-35 [56] and MASS v7.3.47 [57]. Data was checked for normality and transformed where necessary using the Box-Cox routine. The data was $\log$ transformed where the $95 \%$ confidence interval of lambda includes 0 and transformed with $\left(x^{\lambda}-1\right) / \lambda$ in other cases. The significance of the overall differences between the different doses obtained from the various treatments was assessed by ANOVA [58]. The significance of differences between the group means (different radiation doses vs. unirradiated individually analyzed for each day post emergence and irradiation time) was determined by Tukey's honestly significant difference (HSD) test. The $P$-values were calculated from the data with the significance threshold selected as 0.05 (Additional file 2). All regression analyses were conducted using the linear model for different times and different doses and coefficient factors (slope), $\mathrm{t}$ and $P$ values are presented for females and males in Additional files 3 and 4 respectively.

\section{Results \\ Dynamics of Sodalis copy number in non-irradiated G. $m$. morsitans adults}

Experiments carried out under laboratory conditions indicated that Sodalis copy number was correlated with fly age and sex. For both males and females an exponential increase in Sodalis copy number was observed after fly emergence from the puparia, reaching a stable high copy number plateau when flies were aged beyond 3 weeks. In addition, Sodalis copy number was significantly higher in female than male flies $(P<0.001$, regardless of fly age (Fig. 1, Additional file 2).

\section{Impact of irradiation on Sodalis copy number in G. $m$. morsitans}

We evaluated the impact of irradiation on the copy number of Sodalis in adult flies after treatment at three different life stages: (i) as 7-day old adults, (ii) 29-day old puparia and (iii) as 22-day old puparia. Where flies were irradiated as puparia, the analyses were conducted on different days post emergence (dpe), but for flies irradiated as adults the analysis was done on different days post irradiation (dpi).

\section{Adults irradiated at 7-days}

In male flies, the radiation dose and time after irradiation significantly influenced the Sodalis copy number. Sodalis copy number decreased significantly with increasing radiation dose $(P<0.001)$, but increased significantly $(P<0.001$, Fig. 2 , Additional file 2$)$ with time after irradiation. The negative correlation between radiation dose and Sodalis copy number was most obvious on day 


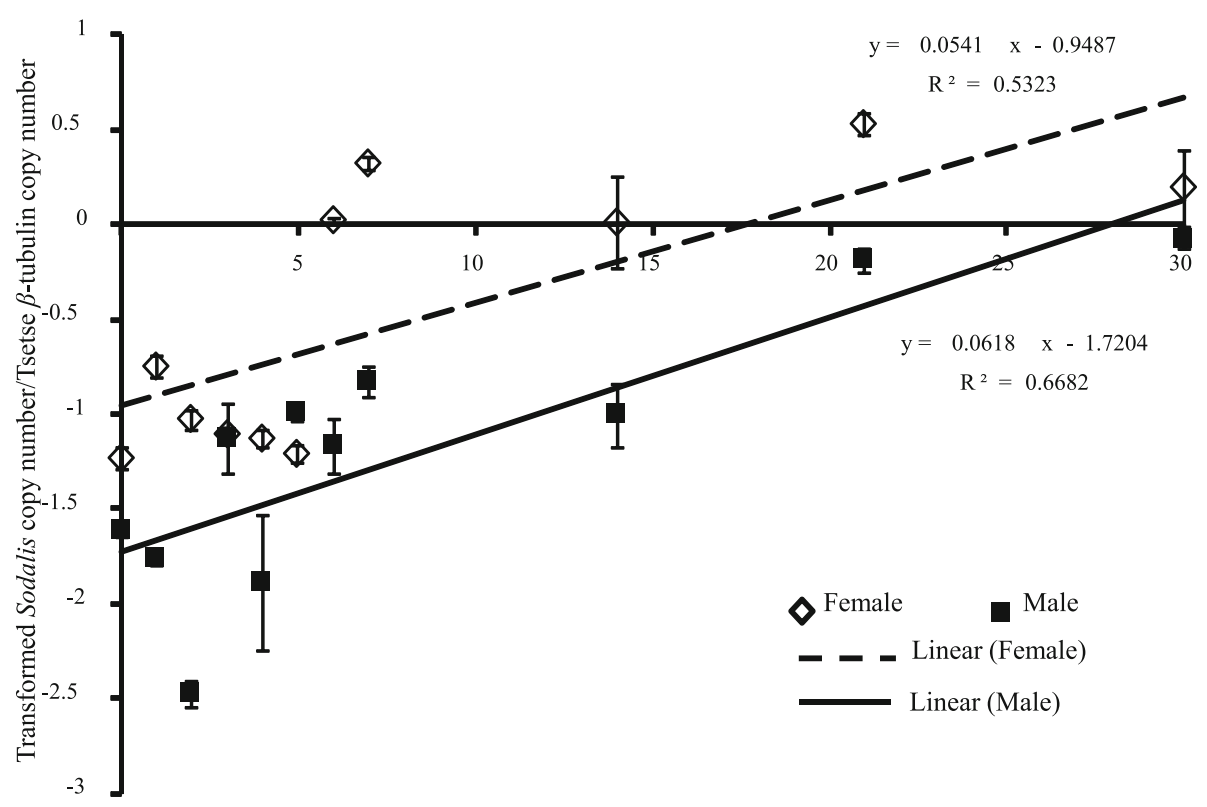

Days post emergence (dpe)

Fig. 1 Dynamics of Sodalis copy number in G. m. morsitans adult flies maintained under laboratory colony conditions. Four males and four females were tested at each time point to estimate the Sodalis copy number using qPCR. Normalized qPCR data were transformed $(\lambda=-0.2)$ to best fit the normal distribution. * shows a significant difference between treatments at different levels (Tukey HSD at the 95\% family wise confidence level), $\left({ }^{*}(P<0.05\right.$ level $\left.),{ }^{* *}(P<0.001),{ }^{* *}(P<0.0001)\right)$

1 and 7 post irradiation. On the day of emergence, no significant impact was observed between the different doses and Sodalis copy number. On day 14 post irradiation, the difference in Sodalis copy number among the different doses was lower than that observed on day 1 and 7 post irradiation but it remained significant (Additional file 3). For the irradiated samples and regardless of the dose, the copy number of Sodalis on day 14 post irradiation was relatively higher than the copy number observed on day 0, 1 and 7 day post irradiation (Fig. 2, Additional file 3). The rate of increase of Sodalis copy number was higher in irradiated samples than nonirradiated controls. In non-irradiated flies, there was no significant regression between Sodalis copy number and time (Additional file 2, Additional file 5A).

The negative impact of radiation dose on Sodalis copy number was lower in females than males; this was mainly obvious on day 7 post irradiation (Fig. 2, Additional file 2). The impact on the Sodalis population following irradiation was most obvious on day 1 post irradiation. This decrease in Sodalis copy number was less obvious but significant on day 14 post irradiation. No significant decrease in Sodalis copy number due to the increase in the dose was observed on day 7 post irradiation. On the day of irradiation, no significant regression between dose and Sodalis copy number was observed (Additional file 3). Over time, there was an increase in Sodalis copy number regardless of the dose. The increase in Sodalis copy number was greater in samples irradiated with 20 and 50 Gy than in 110 Gy and non-irradiated samples (Additional file 4, Additional file 5B).

\section{Adults emerged from 29-day old irradiated puparia}

The impact of irradiation of 29-day old puparia on the Sodalis population was analyzed at different time-points over the course of a 14-day observation period. The irradiation significantly reduced Sodalis copy number in males $(P<0.001)$ (Fig. 3) irrespective of the day after emergence; however, Sodalis copy number significantly increased with time after emergence during the test period regardless of the dose $(P<0.001)$ (Fig. 3, Additional file 2). Sodalis copy number was inversely correlated with the radiation dose and was most obvious on days 1, 3 and 5 post emergence (Fig. 3, Additional file 3). Although Sodalis copy number was lower in irradiated males than control flies regardless of time, the increase in Sodalis copy number over time was higher in irradiated samples compared to control. The rate of increase in Sodalis copy number was higher in samples treated with 50 and 110 Gy as compared with 20 Gy. In non-irradiated samples, Sodalis copy number did not increase with time (Additional file 4, Additional file 6A).

In females, Sodalis copy number decreased significantly with increasing irradiation dose, on days 1,3 , and 5 post emergence. Sodalis copy number was negatively correlated with radiation dose on days $0,1,3,5,7$ and 
Days post irradiation (dpi)

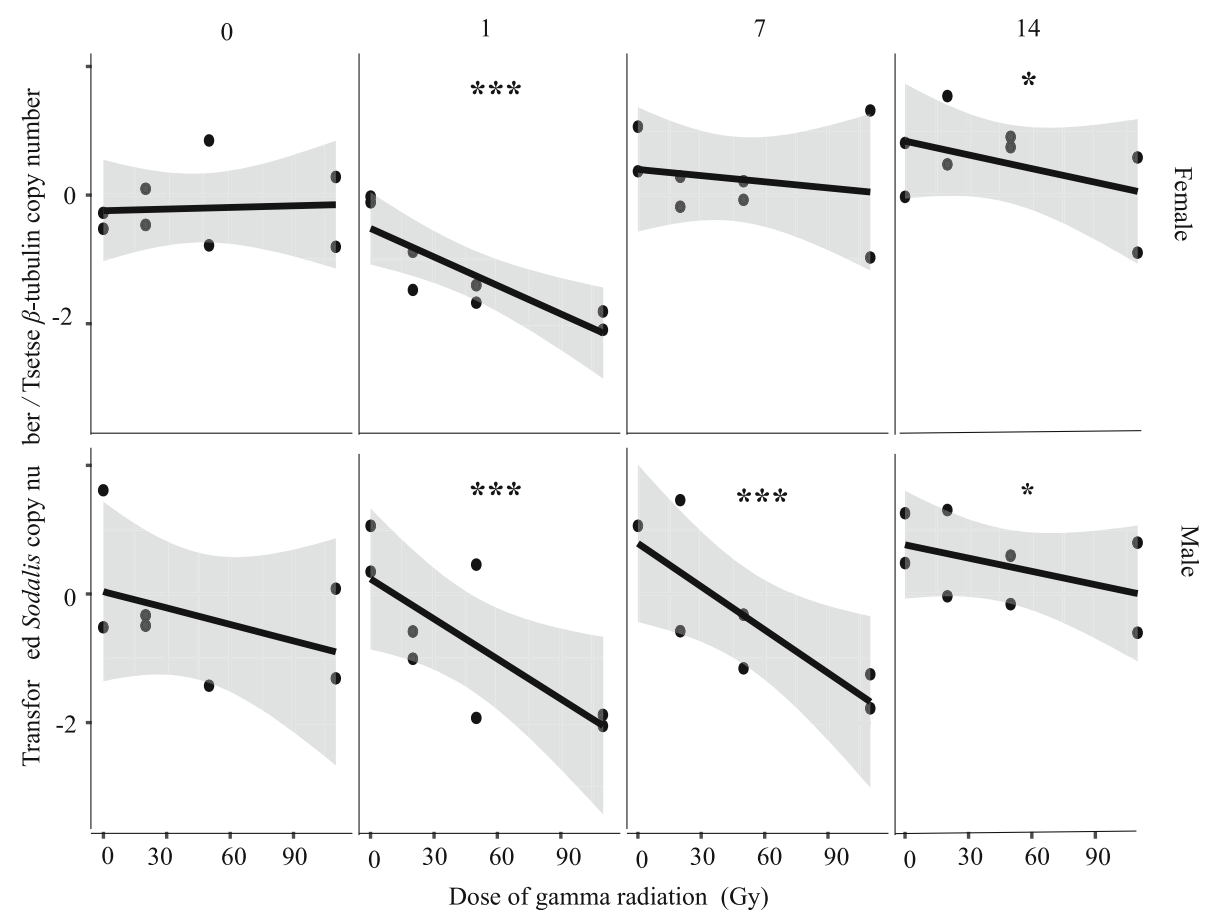

Fig. 2 Impact of different ionizing radiation doses on Sodalis copy number in G. m. morsitans adult flies irradiated at 7-days post emergence at different times post irradiation. Four male and four female 7-day old adults exposed to different radiation doses were used to quantify Sodalis copy number at different time points post-irradiation. Normalized qPCR data were transformed $(\lambda=0.2)$ to best fit the statistical normal distribution. ${ }^{*}$ indicates a significant different between treatments at different levels (Tukey HSD at the $95 \%$ family wise confidence level), ${ }^{*}(P<$ 0.05 level), ${ }^{* *}(P<0.001)$, $\left.{ }^{* *}(P<0.0001)\right)$

14 post emergence (Fig. 3, Additional file 3). Although the copy number of Sodalis in irradiated treatments was in general lower than the non-irradiated control as observed in males, an exception was found at day 14 post emergence, where Sodalis copy number was slightly higher than the control. In the irradiated samples, the lowest Sodalis copy number was found in the samples treated with 110 Gy except on day 7 post emergence, where the lowest copy number was observed in 50 Gy-irradiated samples. As in males, there was a significant positive regression between Sodalis copy number and time post emergence $(P<0.01)$ in the female samples irradiated at 110 Gy (Fig. 2B, Additional file 4). The rate of increase in Sodalis copy number was higher in the 110 Gy irradiated samples as compared with that in 20 and 50 Gy irradiated samples. Surprisingly a significant decrease in Sodalis copy number over time was observed in non-irradiated samples $(P=0.011)$ (Additional file 4, Additional file 6B).

\section{Adults emerged from 22-day old irradiated pupae}

The quantification of Sodalis copy number in adult flies (males and females) emerged from puparia irradiated at 22-days old showed a different profile from that observed in flies irradiated as adults or as 29-day old puparia. However, day post emergence and sex significantly affected Sodalis copy number whilst Sodalis copy number in general was independent of radiation dose (Fig. 4). As there was a significant interaction between time and treatment $(P=0.017)$ and between sex and time $(P<0.01)$ and treatment, the data for each time were analyzed separately for males and females (Additional file 2).

In male flies, Sodalis copy number was in general not affected by dose, but was significantly affected by day post emergence when all data was analyzed together (Fig. 4, Additional file 2). However, when analyzed on each day post emergence, increasing doses induced a decrease in Sodalis copy number on day $0,1,3$ and 7 post emergence. In contrast, Sodalis copy number increased with increasing radiation dose on day 5 and 14 post emergence (Fig. 4, Additional file 3). The rate of increase of Sodalis copy number in non-irradiated controls was not significant with time but was significant for the 20, 50 and 110 Gy-treatment groups (Additional file 4, Additional file 7A).

In general, the copy number of Sodalis was higher in female than in male flies and was independent of radiation dose, but increased significantly with time (Fig. 4, Additional file 2. However, the rate of increase of Sodalis copy number 


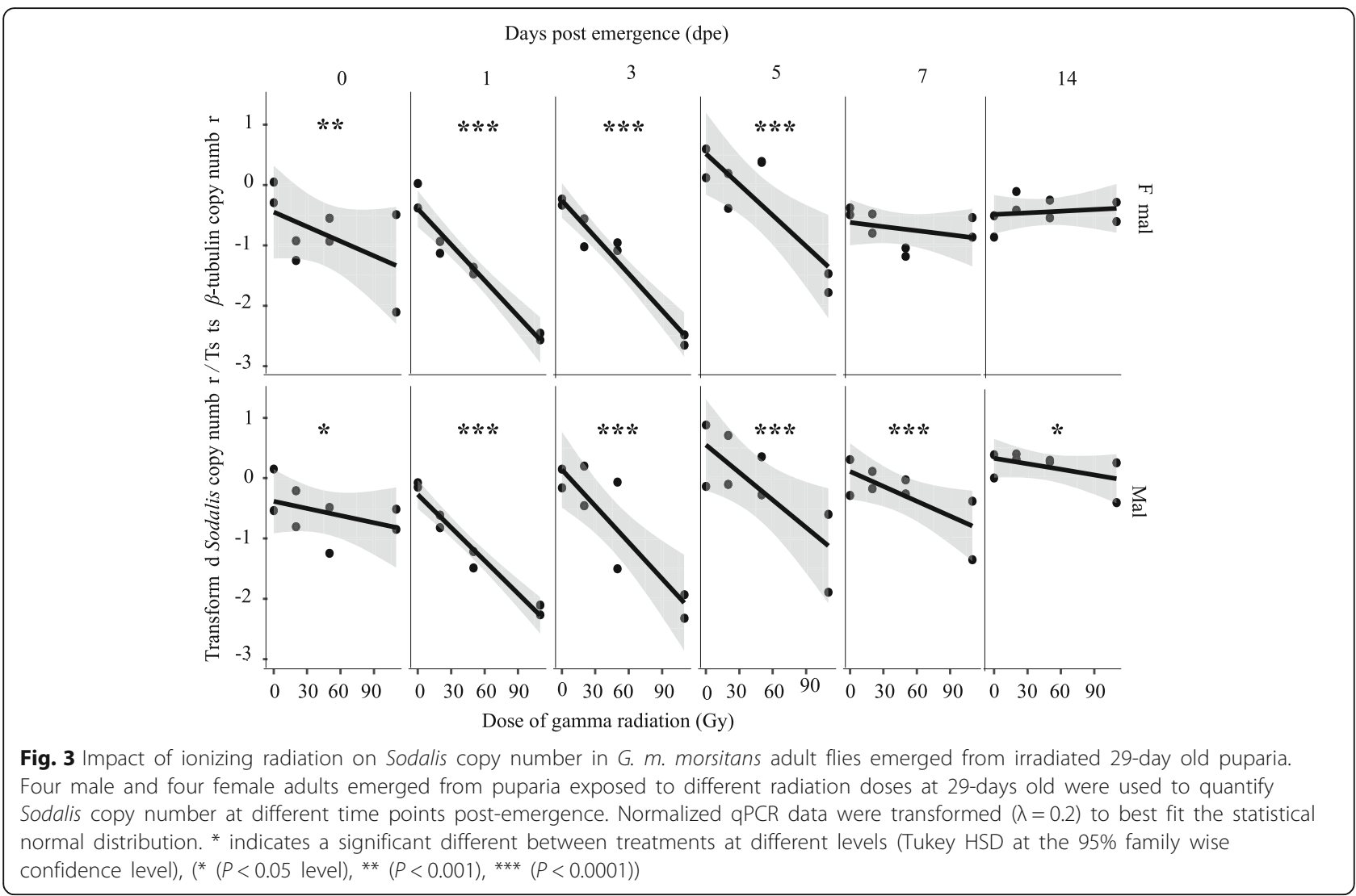

with time in the samples irradiated with 20 and $110 \mathrm{~Gy}$ was much higher than the rate of increase in the samples irradiated with 50 Gy and non-irradiated controls (Additional file 2, Additional file 7B).

\section{Impact of tsetse developmental stage during irradiation on Sodalis copy number in G. m. morsitans males}

Comparing the Sodalis copy number in the nonirradiated control with that in males irradiated with 110 Gy on day 22 and 29 post larviposition and adults, indicated that on day 7 post emergence, the Sodalis copy number was significantly lower than in non-irradiated males $(P=0.002)$, irrespective of the developmental stage at the time of irradiation. On day 14 post emergence, the copy number of Sodalis in males irradiated with 110 Gy as adult males was significantly lower than non-irradiated males $(P<0.001)$. Sodalis copy number was not significantly different in males emerged from puparia irradiated on day 29 and day 22 post larviposition as compared with non-irradiated control flies. It is worth noting that the highest and lowest copy number of Sodalis was observed in males in the irradiated adult treatment in controls and 110 Gy respectively (Fig. 5).
Impact of irradiation of 22-day old puparia on Wigglesworthia and Wolbachia copy number in G. $m$. morsitans flies

Wigglesworthia and Wolbachia densities were significantly different in female flies as compared with male flies (Fig. 6 and Additional file 8). In addition, there was a significant interaction between sex and treatment in Wolbachia; therefore, the data for males and females were analyzed separately (Additional file 2).

In males, the ANOVA indicated that increasing irradiation dose and time did not cause significant changes in Wigglesworthia copy number when analyzed separately (Fig. 6, Additional file 2). However, the regression analysis indicated that the copy number of Wigglesworthia was reduced with increasing dose regardless of the time post emergence but this negative regression was only significant on days 0,3 and 14 post emergence (Additional file 3 ). It is important to note that unlike Sodalis, the Wigglesworthia copy number did not significantly change with time in non-irradiated males or males irradiated with $50 \mathrm{~Gy}$. In males irradiated with 20 and 110 Gy the Wigglesworthia copy number decreased significantly with time (Fig. 6, Additional file 4, Additional file 8A). The copy number of Wolbachia in male flies was not significantly affected by the 

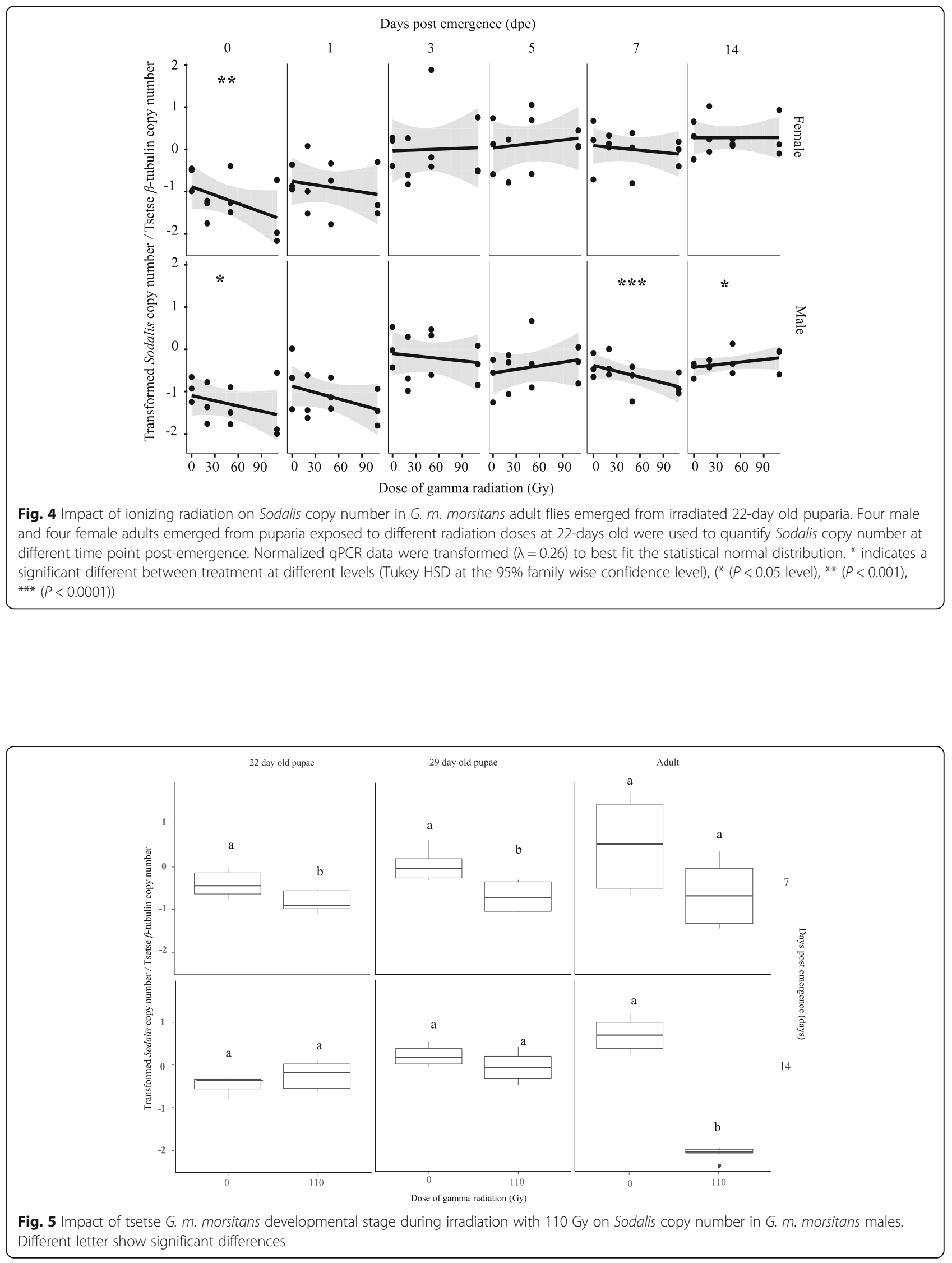


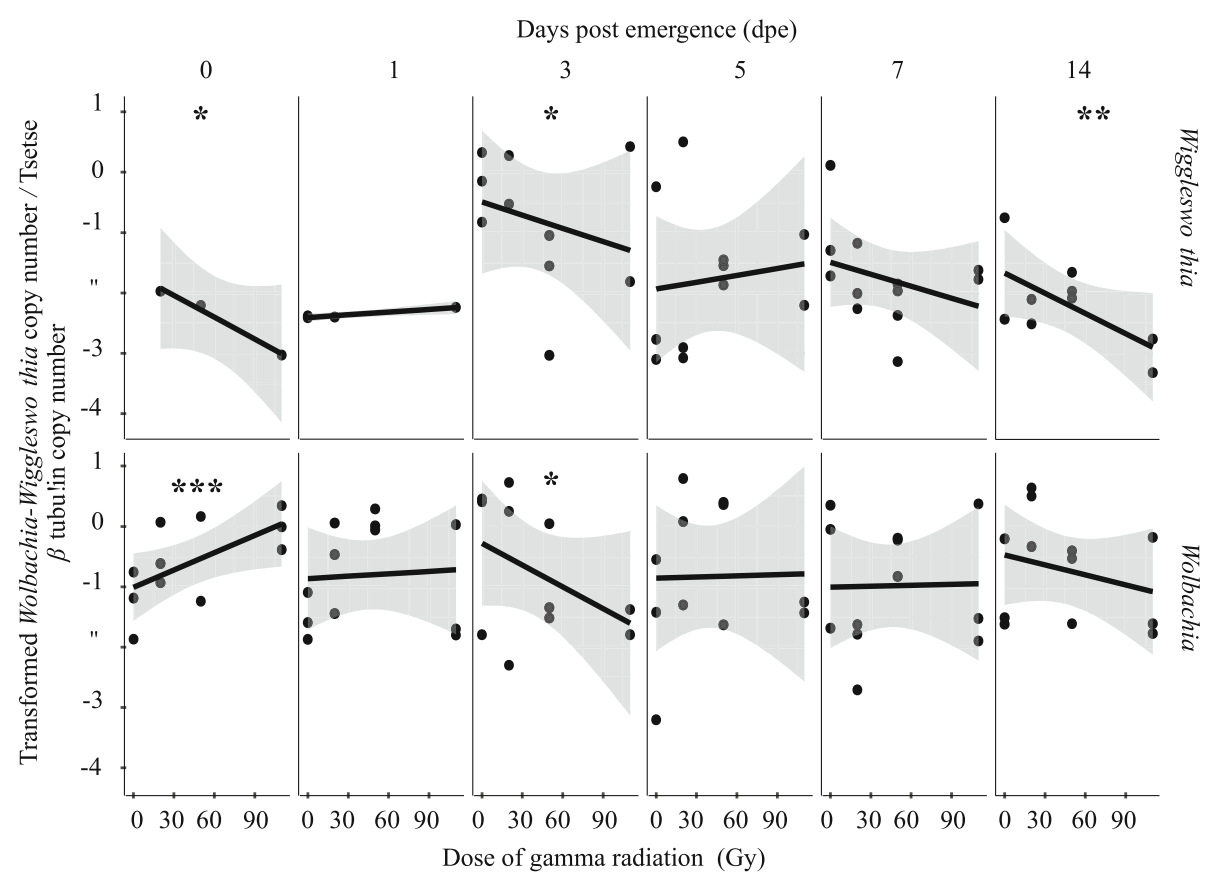

Fig. 6 Impact of ionizing radiation on Wigglesworthia and Wolbachia copy number in G. m. morsitans males. Four male and four female adults emerged from puparia exposed to different radiation doses at 22-days old were used to quantify Wigglesworthia and Wolbachia copy number at different time points post-emergence. Normalized qPCR data were transformed $\lambda=0.02$ and $\lambda=0.2$ ) to best fit the statistical normal distribution. * indicates a significant different between treatments at different levels (Tukey HSD at the 95\% family wise confidence level), $(*(P<0.05$ level), ** $(P<0.001)$, *** $(P<0.0001))$

radiation dose (Additional file 2). Wolbachia copy number increased with increasing dose on the day of emergence. This positive correlation turned into a significant negative correlation on day 3 post emergence (Fig. 6, Additional file 3). The copy number of Wolbachia did not change significantly over time for non-irradiated or irradiated males (Fig. 6, Additional file 2, Additional file 9A).

In female flies, increasing radiation dose or time post emergence did not causes significant changes in the copy number of Wigglesworthia (Fig. 7, and Additional file 2). In general, the copy number of Wigglesworthia was reduced with increasing dose up to day 7 post emergence but seemed to increase with increasing dose on day 14 post emergence (Additional file 3). There was no significant correlation between copy number of Wigglesworthia and time in non-irradiated control flies, whereas significant positive correlation was observed in the 110 Gy treatment group (Additional file 4, Additional file 9A).

Unlike Wigglesworthia, the copy number of Wolbachia in female flies was significantly affected by radiation dose and time post emergence (Fig. 7, Additional file 2). In general, similar to Wigglesworthia the copy number of Wolbachia decreased with increasing doses, with the exception on the day of emergence when the copy number of $\mathrm{Wol}$ bachia increased. The decrease in Wolbachia copy number was significant on days $1,3,5,7$ and 14 post emergence (Additional file 3). Over time, the Wolbachia copy number increased significantly only in 20 Gy treated females (Additional file 4, Additional file 9B).

\section{Impact of irradiation on the tsetse fly's susceptibility towards trypanosomes}

Following per os challenge with trypanosomes in their 1st blood meal, $14.7 \%$ and $6 \%$ of non-irradiated and irradiated G. m. morsitans adults, respectively developed a mature trypanosome infection in their salivary glands (Table 1). At the midgut level a similar infection ratio was observed, i.e. $18 \%$ and $6 \%$ in non-irradiated and irradiated flies, respectively. However, the observed differences in infection rates between irradiated and non-irradiated flies in both the salivary gland and the midgut were not statistically significant (Table 1). Results from this experiment show that the establishment of a trypanosome infection in the tsetse's midgut and the subsequent maturation of this infection were not significantly affected by irradiation.

\section{Discussion}

The implementation of the SIT in the context of an area-wide integrated pest management strategy was successful in eradicating a population of Glossina austeni from Unguja Island of Zanzibar [57]. However, 


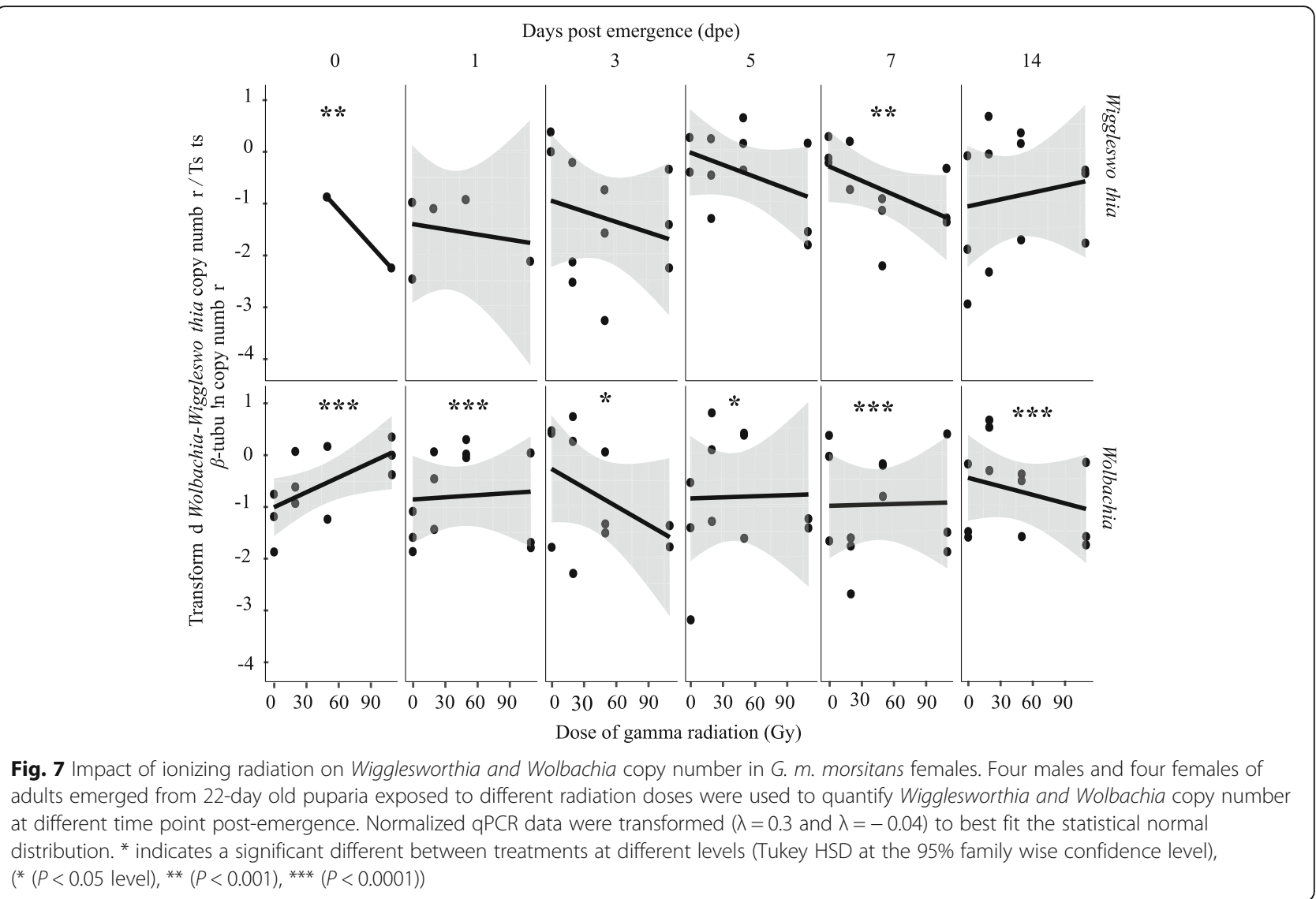

the release of large numbers of sterile male flies bears a potential risk of temporarily increasing disease transmission during the initial release phase of the programme [58]. To date, the release of sterile male tsetse flies has only been implemented in areas without HAT. Before their release, the sterile males are offered blood meals mixed with an anti-trypanosomal drug (isometamidium chloride) and, although this protocol reduced the risk of increased trypanosome transmission, there are reports that claim that it does not completely prevent it $[17,18]$. Therefore, the implementation of a programme with an SIT component in an HAT endemic area will require additional measures to eliminate the risk of increased trypanosome transmission.

One possibility would be to use paratransgenesis to develop tsetse flies refractory to trypanosome infection by exploiting the presense of symbiotic bacteria associated with the flies. It has been suggested to modify the symbiotic bacteria Sodalis to produce anti-trypanosome factors [42, 45, 52, 59] and important recent progress can be reported with the development of paratransgenic tsetse flies [52, 60, 61] for use in SIT programmes [58]. However, as the males destined for release need to be irradiated to make them sterile, the impact of the

Table 1 Light Microscope ${ }^{a}$ evaluation of the proportion of male irradiated and non-irradiated control G. m. morsitans flies infected with T. b. brucei

\begin{tabular}{|c|c|c|c|c|c|}
\hline \multirow[t]{2}{*}{ Treatment } & \multicolumn{2}{|c|}{ Infected/ total \# flies } & \multirow[t]{2}{*}{ Maturation } & \multicolumn{2}{|l|}{$P$ values } \\
\hline & Midgut glands & Salivary gland rate & & Midgut glands & Salivary gland rate \\
\hline Non-irradiated & $11 / 61$ & $9 / 61$ & 0.82 & - & - \\
\hline Irradiated & $3 / 50$ & $3 / 50$ & 1 & 0.0839 & 0.219 \\
\hline
\end{tabular}

$p$ values were obtained by comparing the infection prevalence of the irradiated group to the infection prevalence of non-treated control flies using a two-sided Fisher's exact test.

aLight-Microscope observations/evaluations conducted using 100× magnification to carry out the observations

${ }^{\mathrm{b}}$ Maturation rate: salivary gland/midgut infected flies 
irradiation treatment on the Sodalis community needed to be assessed. Therefore, we investigated the effect of different radiation doses administered during different life stages on the copy number of Sodalis in G. m. morsitans flies.

It is known that the SIT becomes more effective when only males are released, but separating tsetse male from female puparia is currently not possible at an operational scale. In operational SIT programmes implemented so far, tsetse fly males have been separated from females using one the following methods: (i) manual separation of the adults based on the morphological differences, or (ii) exploiting the difference in pupal period (females emerge 2 days earlier than males) [62, 63]. A third method is based on the use of near infrared light [46] to separate the puparia 8-10 days before adults emergence, but this is still under development. The above mentioned methods offer opportunities to irradiate male flies as adults (method 1) or pupae (methods 2 and 3 ) and to sterilize them for the release in an SIT program. Hence, the importance of analysing the impact of irradiation on tsetse symbionts at these different developmental phases. The selected male separation method depends on the conditions of each SIT program: (1) in the program that successfully eradicated a population of G. austeni from Unguja Island of Zanzibar [57], adult males were separated manually from adult females and the males irradiated and released as adults. A similar strategy was used for the programme against Glossina palpalis gambiensis and Glossina tachinoides in Sidéradougou, Burkina Faso [64] and against G. fuscipes fuscipes and G. pallidipes in Ethiopia [65]. A different approach was adopted in the pilot SIT programme against Glossina morsitans in Tanzania, where the flies were irradiated and released as pupae in release stations [66]. Another approach was adopted in the program in Senegal against G. p. gambiensis where the male puparia were collected on day 29 post larviposition after the emergence of females, irradiated and shipped under chilled conditions at $10{ }^{\circ} \mathrm{C}$ from several countries to Dakar, Senegal [67]. Upon arrival, the pupae were left to emerge and the male flies were released as adults in the target area. In the latter case, it is important to point out that separating male and female puparia during the mid-pupal phase (between days 1525 post larviposition) would be much appreciated in SIT programs as it would allow the irradiation and shipment of male puparia under ideal environmental condions (e.g. $23{ }^{\circ} \mathrm{C}$ ), which would result in better quality males. With insects like the Mediterranean fruit fly Ceratitis capitata, the problem was solved through the development of genetic sexing strains (GSS), which allows the females to be eliminated at the embryonic or pupal stage. This approach greatly increased the efficacy of SIT programmes against this pest and significantly reduced its cost $[68,69]$. Unfortunately such an approach is not available for tsetse flies.

The use of ionizing radiation to sterilize male insects is a simple process that is easy and safe to apply [70]. Radiation causes single- and double-strand breaks in the chromosomes of both somatic and germ line cells [71], resulting in the formation of dominant lethal mutations in eggs and sperm [70]. However, as a result of the irradiation free radicals originating from water radiolysis, mainly $\mathrm{OH}$ free radicals, $\mathrm{H}$ atoms and solvated electrons $\mathrm{e}_{\mathrm{aqu}}$, are formed in the treated insect that interact with intra- or extracellular molecules. The free radicals affect the microbial communities associated with irradiated flies as an indirect effect of irradiation. The negative impact of irradiation on reducing the gut microbiota was previously demonstrated in humans [72], but the impact on the microbiota associated with insects was so far not reported.

The results show that the copy number of Sodalis in untreated male and female G. m. morsitans significantly increased with time. Non irradiated female G. m. morsitans had a higher Sodalis copy number than male flies during a period of 30 days after emergence. This contrasts with earlier work that showed that Sodalis densities in male G. p. gambiensis were always higher than in female flies over a period of 80 days [73], and this difference might be due to a species-specific impact on Sodalis copy number or the size of the analyzed samples $(n=$ 8 ) at each time point. In general, the copy number of Sodalis infection in somatic tissues increased with the age of the fly but varied with species and sex [23]. In addition, our results indicate that the Sodalis population was significantly reduced after irradiation of 7-day old adult males, with no significant recovery on day 14 post irradiation. In contrast, the recovery of Sodalis copy number was significant in adult flies treated as 22 or 29 day-old puparia. The recovery in Sodalis copy number was most prominent in female flies when treated as 29-day old puparia, and in male flies when treated as 22-day old puparia. The observed recovery in Sodalis copy number in adult flies treated as pupae might be due to the relative longer period available for multiplication of Sodalis individuals after irradiation in comparison with the shorter period available in irradiated adult males. It is important to note that Sodalis has a relatively slow growth rate $(\sim 15 \mathrm{~h}$ for cell population doubling times in vitro) and therefore a relatively longer period is needed to increase its copy number in the irradiated host [59].

The recovery of Sodalis copy number in males treated as 22 day-old puparia to similar or even higher levels as observed in non-irradiated males opens the opportunity to use paratransgenesis to develop tsetse strains that are refractory to trypanosome infection. Although this study 
was conducted on non-modified Sodalis, it can at this stage be assumed that the response of modified Sodalis to irradiation would be similar to wild Sodalis, but this will need to be confirmed by further research. In our study both puparia and adult flies were irradiated to estimate the optimal dose and effects on Sodalis copy number, and the results clearly indicate that irradiating adult flies prohibits the use of paratransgenesis to develop tsetse strains that are refractory to trypanosome infection. Therefore, the most effective use of paratransgenesis in SIT programs will be achieved when separating the male from the female puparia on day 22 post larviposition using near infrared light, at least for G. m. morsitans (Fig. 8) [46]. This method, however, is still under development and it is important to note that the successful development and use of paratransgenesis in SIT programs might be species dependent and is most certainly closely linked to an optimization of male and female pupal separation protocols.

The results also indicate a general reduction in the copy number of Wigglesworthia and Wolbachia in irradiated flies, especially when the dose was a high as 110 Gy. Whereas Wigglesworthia has a clear role in tsetse females as it provides vitamins necessary for female fertility [78], the role of Wigglesworthia in males is not clear and therefore we cannot speculate on the impact of a reduction in Wigglesworthia copy number in irradiated males. On the other hand, the reduction in Wolbachia copy number might negatively affect the potential of releasing of Wolbachia infected males to enhance sterile insect applications using the cytoplasmic incompatibility $(\mathrm{CI})$ phenomenon as recenly implemented in mosquitoes [31, 37, 74-76]. The reduction in Wolbachia copy number after gamma radiation treatment was previously reported in Brugia malayi adult worms [77]. On the other hand, an enhancement effect on Sodalis, Wigglesworthia and Wolbachia densities was observed with lower radiation doses.

Tsetse flies are in general refractory to parasite transmission as illustrated by the extremely low natural prevalence of trypanosome-infected tsetse flies $(<0.1 \%$ for $T$. brucei) as well as by the low tsetse fly infection rates that are obtained in optimal experimental laboratory conditions. In the vector competence experiment, the results from adults treated as puparia on day 22 post larviposition show that the establishment of a trypanosome infection in the tsetse's midgut and the subsequent maturation of this infection was not affected by the irradiation. However, the pending research question will be to determine effect of irradiation on the vectorial capacity of adult tsetse flies infected with genetically modified Sodalis expressing trypanosome-interfering molecules.

To date, no previous study has been conducted to assess the effect of ionizing radiation on the tsetse flies' symbiont copy number. This study determined the impact of irradiating puparia and adults of G. m. morsitans on the copy number of Sodalis, Wigglesworthia and Wolbachia. Our data indicate that irradiation does not affect the vectorial capacity of the released sterile males,

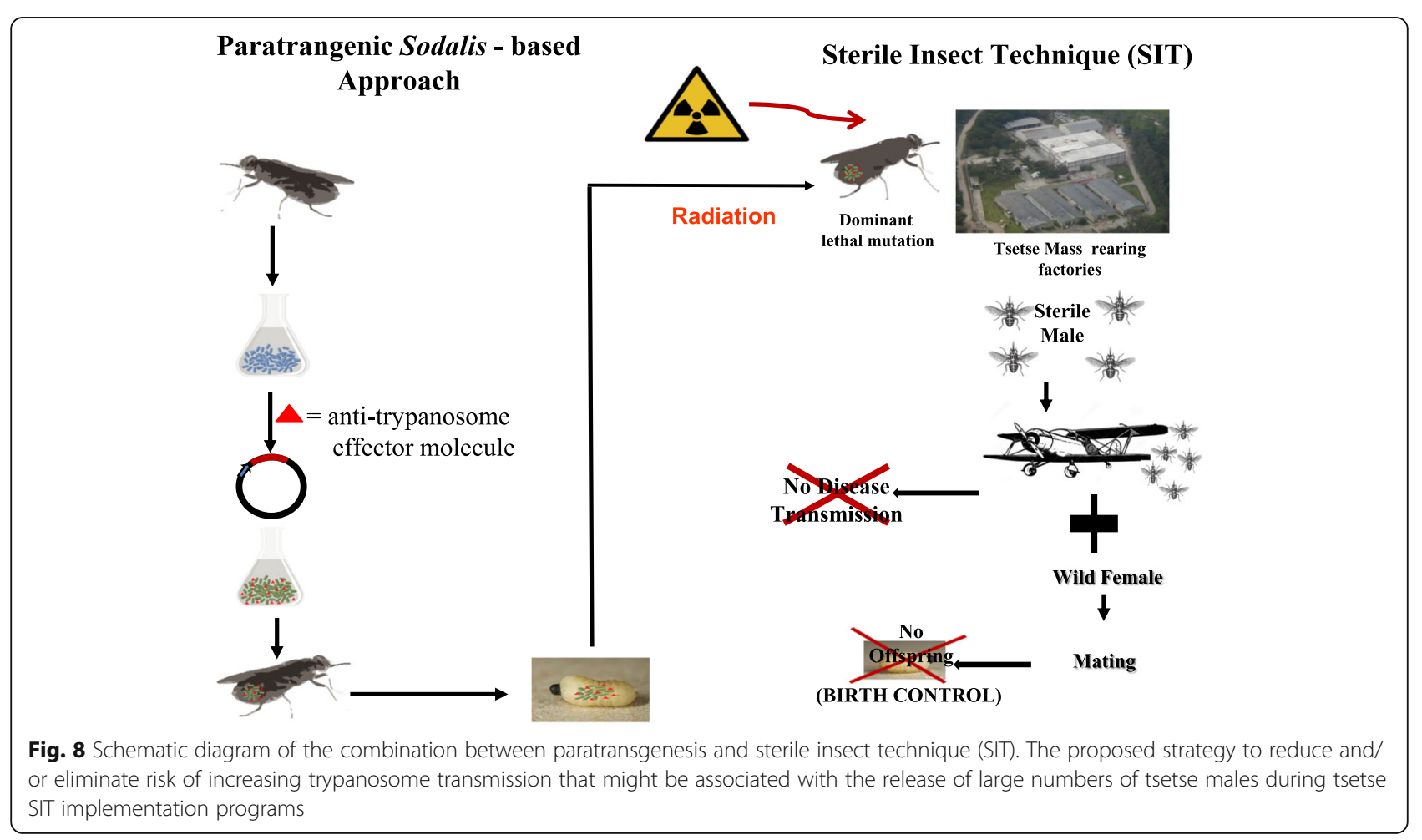


and hence, measures are needed to address this problem. The data of this study are encouraging for the use of paratransgensis to develop strains that are refractory to trypanosome infection, which will reduce or eliminate any potential risk that might be associated with the release of sterile males in HAT endemic areas.

\section{Conclusion}

This study provides the first demonstration of the functional impact of irradiation on Sodalis glossinidus and the vectorial capacity of treated flies. When puparia are irradiated between day 22 and 29 post larviposition, a significant recovery in Sodalis copy number occurs in the adult flies, but the vectorial capacity of adult males is not affected. Moreover, irradiaton induces a significant reduction in the copy number of Wigglesworthia and Wolbachia. The current study also reinforces the idea for the potential use of Sodalis to be developed into a paratransgenic platform that can be combined with SIT to block transmission of trypanosomes.

\section{Additional files}

Additional file 1: List of Primers used for quantitative PCR (qPCR) analyses of microbiome in Glossina species (DOCX $27 \mathrm{~kb}$ )

Additional file 2: ANOVA Statistics for Interaction (DOCX $20 \mathrm{~kb}$ )

Additional file $\mathbf{3}$ Regression Statistics for different time post irradiation (DOCX $28 \mathrm{~kb}$ )

Additional file 4: Regression Statistics for different irradiation doses. (DOCX $25 \mathrm{~kb}$ )

Additional file 5: Impact of time post irradiation on Sodalis copy number in G. m. morsitans adult flies irradiated at 7-day post emergence. Four males (A) and four females (B) of 7-day old adults exposed to different radiation doses were used to quantify Sodalis copy number at different time point post-irradiation foe each irradiation dose. Normalized qPCR data were transformed $(\lambda=0.2)$ to best fit the statistical normal distribution and used for the regression analysis. (PDF $89 \mathrm{~kb}$ )

Additional file 6: Impact of time post irradiation on Sodalis copy number in G. m. morsitans adult flies emerged from irradiated 29-day old puparia. Four males (A) and four females (B) of adults emerged from 29-day old puparia exposed to different radiation doses were used to quantify Sodalis copy number at different time point post-irradiation foe each irradiation dose. Normalized GPCR data were transformed $((\lambda=0.2)$ to best fit the statistical normal distribution and used for the regression analysis. (PDF $95 \mathrm{~kb}$ )

Additional file 7: Impact of time post irradiation on Sodalis copy number in G. m. morsitans adult flies emerged from irradiated 22-day old puparia. Four males (A) and four females (B) of adults emerged from 22-day old puparia exposed to different radiation doses were used to quantify Sodalis copy number at different time point post-irradiation foe each irradiation dose. Normalized qPCR data were transformed $(\lambda=0.26)$ to best fit the statistical normal distribution and used for the regression analysis. (PDF $103 \mathrm{~kb}$ )

Additional file 8: Impact of time post irradiation on Wigglesworthia and Wolbachia copy number in G. m. morsitans adult flies emerged from irradiated 22-day old puparia. Four males of adults emerged from 22-day old puparia exposed to different radiation doses were used to quantify on Wigglesworthia male (A) and Wigglesworthia female (B) copy number at different time point post-irradiation for each irradiation dose. Normalized GPCR data were transformed $\lambda=0.02$ and $\lambda=0.3$ for males and females respectively) to best fit the statistical normal distribution and used for the regression analysis. (PDF $99 \mathrm{~kb}$ )

Additional file 9: Impact of time post irradiation on Wigglesworthia and Wolbachia copy number in G. m. morsitans adult flies emerged from irradiated 22-day old puparia. Four females of adults emerged from 22-day old puparia exposed to different radiation doses were used to quantify on Wolbachia male (A) and Wolbachia female (B) copy number at different time point post-irradiation for each irradiation dose. Normalized GPCR data were transformed ( $\lambda=0.2$ and $\lambda=-0.04$ for males and females respectively) to best fit the statistical normal distribution and used for the regression analysis. (PDF $127 \mathrm{~kb}$ )

\section{Abbreviations}

AAT: Animal African Trypanosomosis; AW-IPM: Area-wide integrated pest management programs; DNA: Deoxyribonucleic acid; dpe: Days post emergence; dpi: Days post irradiation; GSS: Genetic sexing strains; HAT: Human African Trypanosomosis; HSD: Tukey's honesty significant difference test; IPCL: Insect pest control laboratories; ITM: Institute of Tropical Medicine; GPCR: Quantitative polymerase chain reaction; RH: Relative humidity; SIT: Sterile insect technique

\section{Acknowledgments}

The authors acknowledge Carmen Marin, Abdul Hasim Mohamed, Edgardo Lapiz and Henry Adun of the Joint FAO/IAEA Programme, Seibersdorf, Austria, for rearing the experimental flies used in this study.

\section{Funding}

This work and the publication fees were funded by the Joint FAO/IAEA Division of Nuclear Techniques in Food and Agriculture, IAEA (CRP No: D4.20.15) Vienna, Austria.

\section{Availability of data and materials}

The dataset supporting the conclusions of this article is available in the following link https://dataverse.harvard.edu/ dataset.xhtml?persistentld=doi:10.7910/DVN/L6UMML.

\section{About this supplement}

This article has been published as part of BMC Microbiology Volume 18 Supplement 1, 2018: Enhancing Vector Refractoriness to Trypanosome Infection. The full contents of the supplement are available online at https:// bmcmicrobiol.biomedcentral.com/articles/supplements/volume-18supplement-1.

\section{Authors' contributions}

AMMA, AGP, RLM: designed, supervised the research and writing of the manuscript. GDU, LDV, AGP, AMMA: conducted the experiments, collected and analyzed data and prepared the figs. GDU, LDV, AGP, MJBV, JVDA, RLM AMMA: participated in the writing of the manuscript. All authors have read and agreed to its final version.

\section{Ethics approval and consent to participate}

Not applicable.

\section{Consent for publication}

Not applicable.

\section{Competing interests}

The authors declare that they have no competing interests.

\section{Publisher's Note}

Springer Nature remains neutral with regard to jurisdictional claims in published maps and institutional affiliations.

\section{Author details}

'Insect Pest Control Laboratory, Joint FAO/IAEA Division of Nuclear Techniques in Food and Agriculture, Vienna International Centre, P.O. Box 100, 1400 Vienna, Austria. ${ }^{2}$ Institute of Chemical, Environmental, and Biological Engineering, Research Area Biochemical Technology, Vienna University of Technology, Gumpendorfer Straße 1a, 1060 Vienna, Austria. 
${ }^{3}$ Department of Biomedical Sciences, Unit of Veterinary Protozoology, Institute of Tropical Medicine Antwerp (ITM), Antwerp, Belgium.

\section{Published: 23 November 2018}

\section{References}

1. Simarro PP, Franco JR, Cecchi G, Paone M, Diarra A, Ruiz Postigo JA, et al. Human African trypanosomiasis in non-endemic countries (2000-2010)2. J Travel Med. 2012:19:44-53.

2. Welburn SC, Fèvre EM, Coleman PG, Odiit M, Maudlin I. Sleeping sickness: a tale of two dieases. Trends Parasitol. 2001:17:19-24.

3. Reifenberg JM, Solano P, Duvallet G, Cuisance D, Simpore J, Cuny G. Molecular characterization of trypanosome isolates from naturally infected domestic animals in Burkina Faso. Vet Parasitol. 1997;71:251-62.

4. Van Den Abbeele J, Claes Y, Van Bockstaele D, Le Ray D, Coosemans M. Trypanosoma brucei spp. development in the tsetse fly: characterization of the post-mesocyclic stages in the foregut and proboscis. Parasitology. 1999; 118:469-478.

5. Aksoy S. Control of tsetse flies and trypanosomes using molecular genetics. Vet Parasitol. 2003:115:125-45.

6. Butler D. Tropical diseases: raiding the medicine cabinet: Nature Publishing Group; 2003

7. Docampo R, Moreno SN. Current chemotherapy of human African trypanosomiasis. Parasitol Res. 2003;90:510-3.

8. Anene BM, Onah DN, Nawa Y. Drug resistance in pathogenic African trypanosomes: what hopes for the future? Vet Parasitol. 2001:96:83-100.

9. Bouyer J, Seck MT, Sall B, Ndiaye EY, Guerrini L, Vreysen MJB. Stratified entomological sampling in preparation for an area-wide integrated pest management program: the example of Glossina palpalis gambiensis (Diptera: Glossinidae) in the Niayes of Senegal. J Med Entomol. 2010;47:543-52.

10. Jordan AM. Trypanosomiasis control and African rural development. London: Longman; 1986.

11. Leak SGA. Tsetse biology and ecology: their role in the epidemiology and control of trypanosomosis. Wallingford: CABI Publishing; 1998.

12. Brun R, Blum J, Chappuis F, Burri C. Human African trypanosomiasis. Lancet Br Ed. 2010;375:148-59.

13. Vreysen MJB, Seck MT, Sall B, Bouyer J. Tsetse flies: their biology and control using area-wide integrated pest management approaches. J Invertebr Pathol. 2013;112:S15-25.

14. Knipling EF. Sterile-male method of population control. Science. 1959;130: 902-4.

15. Dyck VA, Hendrichs JP, Robinson AS. The Sterile Insect Technique: Principles and Practice in Area-Wide Integrated Pest Management. Dordrecht: Springer; 2005

16. Vreysen MJB, Robinson AS. lonising radiation and area-wide management of insect pests to promote sustainable agriculture. A review. Agron Sustain Dev. 2011;31:233-50.

17. van den Bossche P, Akoda K, Djagmah B, Marcotty T, De Deken R, Kubi C, et al. Effect of isometamidium chloride treatment on susceptibility of tsetse flies (Diptera: Glossinidae) to trypanosome infections. J Med Entomol. 2006; 43:564-7.

18. Bouyer J. Does isometamidium chloride treatment protect tsetse flies from trypanosome infections during SIT campaigns? Med Vet Entomol. 2008;22:140-3.

19. Aksoy S. Tsetse- A haven for microorganisms. Parasitol Today. 2000;16:114-8.

20. Doudoumis V, Blow F, Saridaki A, Augustinos AA, Dyer NA, Goodhead IB, et al. Challenging the Wigglesworthia, Sodalis, Wolbachia symbiosis dogma in tsetse flies: Spiroplasma is present in both laboratory and natural populations. Sci. Rep. 2017.

21. Pais R, Lohs C, Wu Y, Wang JW, Aksoy S. The obligate mutualist Wigglesworthia glossinidia influences reproduction, digestion, and immunity processes of its host, the tsetse fly. Appl Environ Microbiol. 2008;74:5965-74.

22. Attardo GM, Lohs C, Heddi A, Alam UH, Yildirim S, Aksoy S. Analysis of milk gland structure and function in Glossina morsitans: Milk protein production, symbiont populations and fecundity. J Insect Physiol. 2008:54:1236-42.

23. Cheng Q, Aksoy S. Tissue tropism, transmission and expression of foreign genes in vivo in midgut symbionts of tsetse flies. Insect Mo Biol. 1999:8:125-32.

24. Weiss B, Aksoy S. Microbiome influences on insect host vector competence. Trends Parasitol. 2011;27:514-22.
25. Weiss BL, Maltz M, Aksoy S. Obligate symbionts activate immune system development in the tsetse fly. J Immunol. 2012;188:3395-403.

26. Dale C, Welburn SC. The endosymbionts of tsetse flies: manipulating hostparasite interactions. Int J Parasitol. 2001:31:628-31.

27. Farikou O, Njiokou F, Mbida Mbida JA, Njitchouang GR, Djeunga HN, Asonganyi T, et al. Tripartite interactions between tsetse flies, Sodalis glossinidius and trypanosomes - an epidemiological approach in two historical human african trypanosomiasis foci in Cameroon. Infect Genet Evol. 2010;10:115-21.

28. Geiger A, Cuny G, Frutos R. Two tsetse fly species, Glossina palpalis gambiensis and Glossina morsitans morsitans, carry genetically distinct populations of the secondary symbiont Sodalis glossinidius. Appl Environ Microbiol. 2005;71:8941-3.

29. Lindh JM, Lehane MJ. The tsetse fly Glossina fuscipes fuscipes (Diptera: Glossina) harbours a surprising diversity of bacteria other than symbionts. Antonie Van Leeuwenhoek. 2011:99:711-20.

30. Maudlin I, Welburn SC, Mehlitz D. The relationship between rickettsia-likeorganisms and trypanosome infections in natural populations of tsetse in Liberia. Trop Med Parasitol. 1990;41:265-7.

31. Alam U, Medlok J, Brelsfoard C, Pais R, Lohs C, Balmand S, et al. Wolbachiasymbiont infections induce strong cytoplasmic incompatibility in the tsetse flyGlossina morsitans. PLoS Pathog. 2011;7:e1002415.

32. Doudoumis V, Tsiamis G, Wamwiri F, Brelsfoard C, Alam U, Aksoy E, et al. Detection and characterization of Wolbachia infections in laboratory and natural populations of different species of tsetse flies (genus Glossina). BMC Micobiology. 2012;12:S3.

33. Bastian FO, Elzer PH, Wu X. Spiroplasma spp. biofilm formation is instrumental for their role in the pathogenesis of plant, insect and animal diseases. Exp Mol Pathol. 2012;93:116-28.

34. Gasparich GE, Whitcomb RF, Dodge D, French FE, Glass J, Williamson DL. The genus Spiroplasma and its non-helical descendants: phylogenetic classification, correlation with phenotype and roots of the Mycoplasma mycoides clade. Int J Syst Evol Microbiol. 2004;54:893-918.

35. Abd-Alla AMM, Bergoin M, Parker A, Maniania NK, Vlak JM, Bourtzis K, et al. Improving sterile insect technique (SIT) for tsetse flies through research on their symbionts and pathogens. J Invertebr Pathol. 2013;112:S2-10.

36. Anbutsu H, Fukatsu T. Population dynamics of male-killing and non-malekilling spiroplasmas in Drosophila melanogaster. Appl Env Microbiol. 2003;69: 1428-34.

37. Bourtzis K, Lees RS, Hendrichs J, Vreysen MJB. More than one rabbit out of the hat: Radiation, transgenic and symbiont-based approaches for sustainable management of mosquito and tsetse fly populations. Acta Trop. 2016;157:115-30.

38. Wang J, Brelsfoard C, Wu Y, Aksoy S. Intercommunity effects on microbiome and GpSGHV density regulation in tsetse flies. J Invertebr Pathol. 2013;112:S32-9.

39. Beaty BJ. Genetic manipulation of vectors: a potential novel approach for control of vector-borne diseases. Proc Natl Acad Sci. 2000;97:10295-7.

40. Coutinho-Abreu IV, Zhu KY, Ramalho-Ortigao M. Transgenesis and paratransgenesis to control insect-borne diseases: current status and future challenges. Parasitol Int. 2010;59:1-8.

41. Aksoy S, Maudlin I, Dale C, Robinson AS, O'Neill SL. Prospects for control of African trypanosomiasis by tsetse vector manipulation. Trends Parasitol. 2001;17:29-35

42. Beard C, O'Neill S, Mason P, Mandelco L, Woese C, Tesh R, et al. Genetic transformation and phylogeny of bacterial symbionts from tsetse. Insect Mol Biol. 1993:1:123-31.

43. Rio RV, Hu Y, Aksoy S. Strategies of the home-team: symbioses exploited for vector-borne disease control. Trends Microbiol. 2004;12:325-36.

44. Welburn SC, Maudlin I, Ellis DS. In vitro cultivation of rickettsia-likeorganisms from Glossina spp. Ann Trop Med Parasitol. 1987:81:331-5.

45. De Vooght L, Caljon G, Stijlemans B, de Beatselier P, Coosemans M, Van Den Abbeele J. Expression and extracellular release of a functional antitrypanosome Nanobody (R) inSodalis glossinidius, a bacterial symbiont of the tsetse fly. Microb Cell Factories. 2012;11:23

46. Moran ZR, Parker AG. Near infrared imaging as a method of studying tsetse fly (Diptera: Glossinidae) pupal development. J Insect Sci. 2016;16:72.

47. Feldmann U. Guidelines for the rearing of tsetse flies using the membrane feeding technique. In: Ochieng'-Odero JPR, editor. 1st ed. Nairobi: ICIPE Science Press: 1994. p. 449-71.

48. Gooding RH, Feldmann U, Robinson AS. Care and maintenance of tsetse colonies. In: Crampton JM, Beard CB, Louis C, editors. . London: Chapman \& Hall Ltd.; 1997. p. 41-55. 
49. R Core Team. R: A Language and Environment for Statistical Computing. Vienna: R Foundation for Statistical Computing; 2017. Available from: https://www.R-project.org/

50. RStudio Team. RStudio: Integrated Development Environment for R [Internet] Boston: RStudio, Inc.; 2015. Available from: http://www.rstudio.com/

51. Le Ray D, Barry J, Easton C, Vickerman K. First tsetse fly transmission of the "AnTat" serodeme of Trypanosoma brucei. Ann Soc Belg Med Trop. 1977;57: 369-81.

52. Caljon G, Van Reet N, De Trez C, Vermeersch M, Pérez-Morga D, Van Den Abbeele J. The dermis as a delivery site of Trypanosoma brucei for tsetse flies. PLoS Pathog. 2016;12:e1005744.

53. Wickham H. ggplot2: elegant graphics for data analysis. New York: Springer; 2009.

54. Sarkar D. Lattice: Multivariate Data Visualization with R. New York: Springer; 2008. Available from: http://Imdvr.r-forge.r-project.org

55. Venables WN, Ripley BD. Modern Applied Statistics with S. Fourth ed. New York: Springer; 2002. Available from: http://www.stats.ox.ac.uk/pub/MASS4

56. Sokal RR, Rohlf FJ. Biometry: the principles and practice of statistics in biological research. New York: Freeman and Company; 1995.

57. Vreysen MJB, Saleh KM, Ali MY, Abdulla AM, Zhu Z-R, Juma KG, et al. Glossina austeni (Diptera: Glossinidae) eradicated on the island of Unguja, Zanzibar, using the sterile insect technique. J Econ Entomol. 2000;93:123-35.

58. Van Den Abbeele J, Bourtzis K, Weiss B, Cordón-Rosales C, Miller W, AMM A$A$, et al. Enhancing tsetse fly refractoriness to trypanosome infection - a new IAEA coordinated research project. J Invertebr Pathol. 2013;112:S142-7.

59. De Vooght L, Caljon G, De Ridder K, van den Abbeele J. Delivery of a functional anti-trypanosome Nanobody in different tsetse fly tissues via a bacterial symbiont, Sodalis glossinidius. Microb Cell Factories. 2014;13.

60. Caljon G, Hussain S, Vermeiren L, Van Den Abbeele J. Description of a Nanobody-based Competitive Immunoassay to Detect Tsetse Fly Exposure. PLoS Negl Trop Dis. 2015;9:e0003456.

61. De Vooght L, Caljon G, Van Hees J, Van Den Abbeele J. Paternal transmission of a secondary symbiont during mating in the viviparous tsetse fly. Mol Biol Evol. 2015.

62. IAEA. In: IAEA/FAO, editor. Automation for tsetse mass rearing for use in sterile insect technique programmes. Vienna: International Atomic Enegy Agency (IAEA); 2003.

63. Zdarek J, Denlinger DL. Changes in temperature, not photoperiod, control the pattern of adult eclosion in the tsetse, Glossina morsitans. Physiol Entomol. 1995:20:362-6.

64. Politzar $H$, Cuisance D. An integrated campaign against riverine tsetse, Glossina palpalis gambiensis and Glossina tachinoides, by trapping, and the release of sterile males. Insect Sci Its Appl. 1984;5:439-42.

65. Kebede A, Ashenafi H, Daya T. A review on sterile instect technique (SIT) and its potential towards tsetse eradication in Ethiopia. Adv Life Sci Technol. 2015:37:24-44.

66. Williamson DL, Baumgartner HH, Mtuya AG, Gates DB, Cobb PE, Dame DA. Integration of insect sterility and insecticides for control of Glossina morsitans morsitans Westwood (Diptera: Glossinidae) in Tanzania: II. Methods of sterilization, transportation and release of sterilised insects. Bull Entomol Res. 1983;73:267-73.

67. Seck MT, Pagabeleguem S, Bassene MD, Fall AG, Diouf TAR, Sall B, et al. Quality of sterile male tsetse after long distance transport as chilled, irradiated pupae. PLoS Negl Trop Dis. 2015;9:e0004229.

68. Franz G. Genetic sexing strains in Mediterranean fruit fly, an example for other species amenable to large-scale rearing as required for the sterile insect technique. In: Dyck VA, Hendrichs J, Robinson AS, editors. Sterile Insect Tech Princ Pract Area-Wide Integr Pest Manag. Dordrecht: Springer; 2005. p. 427-51

69. Marec F, Neven LG, Robinson AS, Vreysen M, Goldsmith MR, Nagaraju J, et al. Development of genetic sexing strains in Lepidoptera: From traditional to transgenic approaches. J Econ Entomol. 2005;98:248-59.

70. LaChance LE. The induction of dominant lethal mutations in insects by ionizing radiation and chemicals-as related to the sterile male technique of insect control. In: Wright JW, Pal R, editors. . Amsterdam: Elsevier; 1967. p. 617-50.

71. Verde SC, Silva T, Matos P. Effects of gamma radiation on wastewater microbiota. Radiat Environ Biophys. 2016:55:125-31.

72. Nam Y-D, Kim HJ, Seo J-G, Kang SW, Bae J-W. Impact of pelvic radiotherapy on gut microbiota of gynecological cancer patients revealed by massive pyrosequencing. PLoS One. 2013;8:e82659.
73. Soumana IH, Berthier D, Tchicaya B, Thevenon S, Njiokou F, Cuny G, et al. Population dynamics of Glossina palpalis gambiensis symbionts, Sodalis glossinidius, and Wigglesworthia glossinidia, throughout host-fly development. Infect Genet Evol. 2013;13:41-8.

74. Zhang D, Lees RS, Xi Z, Bourtzis K, Gilles JRL. Combining the Sterile Insect Technique with the Incompatible Insect Technique: II-Robust mating competitiveness of irradiated triple Wolbachia-Infected Aedes albopictus males under semi-field conditions. PLoS One. 2016;11:e0151864.

75. Zhang D, Zheng X, Xi Z, Bourtzis K, Gilles JRL. Combining the sterile insect technique with the incompatible insect technique: I-impact of Wolbachia infection on the fitness of triple-and double-infected strains of Aedes albopictus. PLoS One. 2015;10:e0121126.

76. Zhang D, Lees RS, Xi Z, Gilles JRL, Bourtzis K. Combining the sterile insect technique with Wolbachia-based approaches: II-A safer approach to Aedes albopictus population suppression programmes, designed to minimize the consequences of inadvertent female release. PLoS One. 2015;10:e0135194.

77. Rao RU, Atkinson LJ, Vanderwall RP, Weil GJ. Brugia malayi: effects of gamma radiation on adult worms and their intracellular Wolbachia bacteria. Exp Parasitol. 2005;109:87-93.
Ready to submit your research? Choose BMC and benefit from:

- fast, convenient online submission

- thorough peer review by experienced researchers in your field

- rapid publication on acceptance

- support for research data, including large and complex data types

- gold Open Access which fosters wider collaboration and increased citations

- maximum visibility for your research: over $100 \mathrm{M}$ website views per year

At BMC, research is always in progress.

Learn more biomedcentral.com/submissions 